\title{
POLIPOSE ADENOMATOSA FAMILIAR - RELATO DE CASO
}

\author{
Ademar Gonçalves CAIXETA NETO1 \\ Andréa Caixeta GONÇALVES²
}

\begin{abstract}
1-Fisioterapeuta e Nutricionista. Especialista em Fisioterapia Cardiorrespiratória e Terapia Intensiva pelo Centro Universitário do Triângulo (UNITRI). E-mail: ademarcaixeta@yahoo.com.br

2-Fisioterapeuta. Especialista em Fisioterapia Cardiorrespiratória e Terapia Intensiva pelo Centro Universitário do Triângulo (UNITRI). Fisioterapeuta do Hospital de Clínicas da Universidade Federal de Uberlândia (HC-UFU). E-mail: deiacaixeta@hotmail.com
\end{abstract}

\section{Recebido em: 30/10/2013 - Aprovado em: 22/12/2013 - Disponibilizado em: 15/01/2014}

RESUMO: A Polipose Adenomatosa Familiar (PAF) é uma doença hereditária caracterizada pela presença de múltiplos adenomas de cólon. É causada por uma mutação germinativa com herança genética autossômica dominante no gene APC (adenomatous polyposis coli), localizado no cromossomo 5q21. Trata-se de uma enfermidade pouco frequente, cuja prevalência varia entre 1/5000 a 1/7500 casos, sendo responsável por apenas $1 \%$ dos casos de câncer colorretal. O objetivo deste estudo foi reportar o caso de uma paciente com PAF atendidida em um Hospital Público de Belo Horizonte-MG, em relação a evolução nutricional, clínica e bioquímica no período pré e pós-operatório.

Palavras-chave: Polipose Adenomatosa Familiar. Estado nutricional. Pré-Operatório. Pós-Operatório. Dietoterapia.

\begin{abstract}
The Familial Adenomatous Polyposis (FAP) is an inherited disease characterized by the presence of multiple adenomas of the colon and caused by a germline mutation with inherited autosomal dominant in APC gene (adenomatous polyposis coli), located on chromosome 5q21. It is an uncommon disease, whose prevalence varies between 1/5000 a 1/7500 cases, accounting for only $1 \%$ of cases of colorectal cancer. The aim of this study was to report the case of a patient with FAP treated in a Public Hospital from Belo Horizonte-MG, in relation to nutritional, clinical and biochemistry evolution, in the pre and postoperative period.
\end{abstract}

Key-words: Familial Adenomatous Polyposis. Nutrional status. Preoperative. Postoperative. Dietary Treatment.

\section{INTRODUÇÃO}

\section{A Polipose Adenomatosa Familiar}

(PAF) é uma doença hereditária caracterizada pela presença de múltiplos adenomas de cólon (GARCIA et al., 2011).

Esta síndrome é causada por uma mutação germinativa com herança genética autossômica dominante no gene APC (adenomatous polyposis coli), localizado no cromossomo 5q21 (KINZLER et al.,1991).
O APC é um gene que associado à supressão tumoral, uma vez que a proteína por ele codificada promove a adesão de células através de sua ligação à $\beta$-catenina ou a microtúbulos. Redução da adesão e aumento da proliferação celular são consequências de anormalidades do mesmo (ROSA \& COIMBRA, 2009).

Trata-se de uma enfermidade pouco frequente, cuja prevalência varia entre 1/5000 
a 1/7500 casos (Garcia et al., 2011), sendo responsável por apenas $1 \%$ dos casos de câncer colorretal (HABR-GAMA \& CAMPOS, 1994).

A combinação da PAF com manifestações extracolônicas (MEC) é referida de forma bastante comum e é denominada Síndrome de Gardner (GARDNER, 1951).

Em estudo retrospectivo de Campos et al. (2003), as MEC mais comuns em pacientes atendidos no Serviço de Coloproctologia do Hospital das Clínicas da Faculdade de Medicina da Universidade de São Paulo no período de 1977 e 2001 foram: cistos epidermóides, pigmentação da retina, osteomas, tumores desmóides e adenoma gástrico.

O objetivo deste estudo de caso foi relatar as manifestações nutricionais clínicas e bioquímicas pré e pós-operatórias de um paciente com PAF.

\section{ESTUDO DE CASO}

Paciente DSF, 32 anos, sexo feminino, deu entrada no Hospital Júlia Kubistchek no dia 23/03/13 para realização de colectomia total devido à polipose adenomatosa familiar. Nega comorbidades, uso de medicação contínua, alergia e cirurgias prévias. Relata ter realizado cateterismo na infância em função de endocardite infecciosa. Ao exame físico encontrava-se hidrata, corada, acianótica, anictérica e afebril. Dados vitais normais (T: $36,6^{\circ} \mathrm{C}$; FC: $72 \mathrm{bpm}$; FR: 17irpm; PA: 120/80mmHg). A partir da triagem nutricional, a paciente foi classificada como eutrófica $(\mathrm{P}=49 \mathrm{~kg}, \mathrm{~A}=161 \mathrm{~cm}, \mathrm{IMC}=18,9$ $\mathrm{kg} / \mathrm{m}^{2}$ ), sem risco nutricional a princípio, por se tratar de cirurgia eletiva com baixo risco e rápida evolução clínica, a qual possui tempo de internação hospitalar médio de 6 a 11 dias e taxa de complicação variando entre 15 e 20\% (ZAGAR-SHOSTARI \& HILL, 2008).

Em relação aos procedimentos éticos, este estudo de caso acompanhou as normas da Resolução 196/96 do Conselho Nacional de Saúde sobre pesquisa envolvendo seres humanos e foi realizado mediante concordância do paciente, a qual assinou previamente um termo de consentimento livre e esclarecido (TCLE).

Necessidades Energéticas no Pré e PósOperatório Imediato

\section{-Harris Benedict:}

$\mathrm{TMB}=655,1+[1,85 \times \mathrm{A}(\mathrm{cm})]+[9,5634 \mathrm{x}$

$\mathrm{P}(\mathrm{kg})]-[4,6756$ x I(anos) $]$

$\mathrm{TMB}=655,1+[1,85 \times 161]+[9,5634 \times 49]$

$-[4,6756 \times 34]$

$\mathrm{TMB}=1262,58 \mathrm{kcal}$

$\mathrm{GET}=\mathrm{TMB} \times \mathrm{FA} \times \mathrm{FL}$

$\mathrm{GET}=1262,58 \times 1,25 \times 1,1=1736 \mathrm{kcal}$

-Fórmula de Bolso: 
$\mathrm{GET}=\mathrm{P}(\mathrm{kg}) \times 35=49 \times 35=1715 \mathrm{kcal}$

$\mathrm{PTN}=\mathrm{P}(\mathrm{kg}) \times 1,2 \mathrm{~g} / \mathrm{kg}=49 \times 1,2=59 \mathrm{~g} \times 4=$ $236 \mathrm{kcal}$

-Necessidades de Macronutrientes:

Proteínas Lipídeos

1736 kcal -- $100 \% \quad 1736$ kcal -- 100\%

236 kcal -- X $\quad$ Y $\quad--30 \%$

$\mathrm{X}=13,6 \% \quad \mathrm{Y}=521 \mathrm{kcal}(58 \mathrm{~g})$

Carboidratos

1736 kcal -- $100 \%$

W $\quad--56,4 \%$

$\mathrm{W}=979 \mathrm{kcal}(245 \mathrm{~g})$

Necessidades Energéticas no PósOperatório Tardio

$-F A O(1985):$

$\mathrm{TMB}=8,7 \mathrm{P}+829$

$\mathrm{TMB}=8,7 \times 49+829$

$\mathrm{TMB}=1255,3 \mathrm{kcal}$

GET $=$ TMB $\times$ FA

$\mathrm{GET}=1255,3 \times 1,56=1958 \mathrm{kcal}$

$\mathrm{PTN}=\mathrm{P}(\mathrm{kg}) \times 1 \mathrm{~g} / \mathrm{kg}=49 \times 1,2=49 \mathrm{~g} \times 4=$ $236 \mathrm{kcal}$
-Necessidades de Macronutrientes:

Proteínas Lipídeos

1958 kcal -- $100 \% \quad 1958$ kcal -- 100\%

236 kcal -- X $\quad Y \quad--30 \%$

$\mathrm{X}=12 \% \quad \mathrm{Y}=588 \mathrm{kcal}(65 \mathrm{~g})$

Carboidratos

$1958 \mathrm{kcal}--100 \%$

W $\quad--58 \%$

$\mathrm{W}=1136 \mathrm{kcal}(284 \mathrm{~g})$

\section{Adequação de Macronutrientes}

Inicialmente, adotou-se como estratégia dietoterápica a associação de dieta VO livre (850,4 kcal; 118,8g CHO; 14g LIP; 62,3g PTN) com suplemento oral - 2 vezes/dia (600 kcal; 80g CHO; 19,6g LIP; 26g PTN). No entanto, foi possível perceber que houve baixa adequação entre os micronutrientes consumidos em relação ao que foi oferecido $(\mathrm{PTN}=35 \%$; LIP $=21 \%$; $\mathrm{CHO}=30 \%$ ) e principalmente ao que seria necessário $(\mathrm{PTN}=53 \% ; \mathrm{LIP}=12 \% ; \mathrm{CHO}=$ $24 \%$ ), conforme demonstrado na tabela 1 . Tal fato justifica-se pela aceitação parcial da dieta VO $(50 \%)$ constatada a partir de registro alimentar de 3 dias (não consecutivos) e da não aceitação do suplemento contendo lactose, uma vez que a paciente era parcialmente intolerante a este nutriente. 
Tabela 1 - Adequação inicial dos macronutrientes consumidos em relação aos oferecidos e necessários à paciente.

\begin{tabular}{lccc}
\hline Macronutrientes & Consumidos & Oferecidos & Necessários \\
\hline Proteínas & $31,1 \mathrm{~g}$ & $88,3 \mathrm{~g}$ & $59,0 \mathrm{~g}$ \\
Lipídeos & $7,0 \mathrm{~g}$ & $33,6 \mathrm{~g}$ & $58,0 \mathrm{~g}$ \\
Carboidratos & $59,4 \mathrm{~g}$ & $198,8 \mathrm{~g}$ & $245,0 \mathrm{~g}$ \\
\hline
\end{tabular}

Em função disso, optou-se pela modificação do suplemento, feito à base de água, mantendo a mesma concentração de energia e macronutrientes, fator fundamental para melhora da aceitação do suplemento pela paciente e, consequentemente, do aumento da adequação ao que foi oferecido (PTN = 59\%; $\mathrm{LIP}=71 \% ; \mathrm{CHO}=63 \%$ ) e também ao que seria necessário $(\mathrm{PTN}=75 \% ; \mathrm{LIP}=29 \%$; $\mathrm{CHO}=41 \%)$, sobretudo no que diz respeito às proteínas.

Tabela 2 - Adequação final dos macronutrientes consumidos em relação aos oferecidos e necessários à paciente.

\begin{tabular}{lccc}
\hline Macronutrientes & Consumidos & Oferecidos & Necessários \\
\hline Proteínas & $44,1 \mathrm{~g}$ & $75,3 \mathrm{~g}$ & $59,0 \mathrm{~g}$ \\
Lipídeos & $16,8 \mathrm{~g}$ & $23,8 \mathrm{~g}$ & $58,0 \mathrm{~g}$ \\
Carboidratos & $99,4 \mathrm{~g}$ & $158,8 \mathrm{~g}$ & $245,0 \mathrm{~g}$ \\
\hline
\end{tabular}

Quanto ao valor calórico total, houve adequação de $84 \%$ e $66 \%$ em relação à quantidade de calorias oferecidas/necessárias inicialmente e após modificação da composição do suplemento oral, respectivamente. A despeito da redução deste percentual, constatou-se melhora nos valores de calorias oferecidas/consumidas (29\%-63\%) e consumidas/necessárias (25\%-42\%).

\section{EVOLUÇÃO CLÍNICA}

No período pré-operatório, paciente em jejum de 12 horas, com hábitos fisiológicos preservados, negando náuseas e vômitos, encontrava-se eutrófica $(\mathrm{P}=49 \mathrm{~kg}$, $\mathrm{A}=161 \mathrm{~cm}, \mathrm{IMC}=18,9 \mathrm{~kg} / \mathrm{m}^{2}$ ), referindo perda ponderal de $3 \mathrm{~kg}$ em 3 meses associada a apetite/ingestão alimentar reduzidos em função do quadro álgico, não tendo sido 
realizadas avaliações antropométricas anteriores.

No pós-operatório imediato, relatou dor abdominal e negou outros sintomas, em uso de dipirona $(500 \mathrm{mg} / \mathrm{ml})$. Já no PO tardio, estava com dieta suspensa, fezes presentes, hematúria, referiu náuseas sem vômitos.

No $2^{\circ}$ e $3^{\circ} \mathrm{DPO}$, paciente apresentou vômitos, diurese clara, evacuação líquida sem sangue, sendo liberados líquidos, evoluindo para dieta líquida restrita no dia seguinte e sendo iniciados suplementos orais ( $2 \mathrm{x} / \mathrm{dia})$, os quais não foram bem aceitos pela paciente.

No $5^{\circ}$ DPO, vômitos ausentes, em uso de dieta VO branda evoluindo para dieta livre no dia seguinte, sendo mantidos analgésicos e ondasetrona. Foi realizado exame bioquímico que revelou valores normais para $\mathrm{Hb}: 11.5 \mathrm{~g} / \mathrm{dl}$, Ht: $33.3 \%$, VCM: $98 \mu \mathrm{m}^{3}$, Linfócitos: $9.2 \%$.

No $8^{\circ} \mathrm{DPO}$, ileostomia funcionante, fezes pastosas, vômitos recorrentes, utilização fixa de ondasetrona e plasil.

No $9^{\circ}$ e $10^{\circ}$ DPO, evoluindo sem náuseas e vômitos, em uso de dieta VO livre e suplementos orais (HPHC e $10 \mathrm{~g}$ fibra 1x/dia) com aceitação parcial, soroterapia suspensa.

Nos DPO posteriores, evoluiu com hábitos fisiológicos preservados, náuseas e vômitos ausentes, melhora do apetite, média aceitação de dieta VO e boa aceitação dos suplementos orais.

No $14^{\circ}$ DPO, estava estável clinicamente, corada, hidratada, acianótica, anictérica e afebril. Quanto ao estado nutricional permanecia eutrófica com pequeno ganho ponderal em relação ao pré-operatório $\left(\mathrm{P}=49,5 \mathrm{~kg}, \mathrm{~A}=161 \mathrm{~cm}, \mathrm{IMC}=19,1 \mathrm{~kg} / \mathrm{m}^{2}\right)$. No período da tarde recebeu alta hospitalar.

\section{DISCUSSÃO}

O sexo feminino é considerado um fator de risco independente para polipose adenomatosa familiar clássica (PENNA et al., 1993; BERTARIO et al., 2001), que é caracterizada pela presença de centenas de pólipos colorretais adenomatosos que progridem de forma praticamente inevitável para o câncer colorretal em torno de 35 a 40 anos de idade (CAMPOS, 2006), gênero e faixa etária da paciente, motivo pelo qual optou-se pela colectomia total como tratamento preventivo.

Após a identificação de um caso, deve ser realizado o rastreamento de familiares para identificar eventuais portadores do defeito genético, uma vez que filhos de um indivíduo com esta condição têm $50 \%$ de chance de desenvolvê-la (CAMPOS, 2006).

Desta forma, a detecção precoce assume fundamental importância no 
tratamento, uma vez que permite a realização de colectomia profilática, reduzindo a possibilidade de desenvolvimento de câncer posteriormente (CAMPOS, 2006).

\section{TERAPIA NUTRICIONAL}

A dietoterapia no período préoperatório imediato varia em função do órgão ou seguimento envolvido no processo cirúrgico, assim como o tipo de anestesia. São indicadas dietas leves 15 horas antes da operação e jejum de 8 a 12 horas antes, para evitar o acúmulo de resíduos facilitando eventuais vômitos. Naqueles indivíduos com trânsito intestinal lento, deve-se utilizar preferencialmente dieta pobre em alimentos formadores de resíduos, de consistência leve ou líquida, conforme adotado para a paciente em questão. Em casos mais delicados ou de acordo com a demanda, poderiam ser utilizados módulos de nutrientes e/ou suplementos alimentares (BAXTER \& WAITZBERG, 2006).

Na fase pós-operatória imediata devese: contornar as intolerâncias alimentares decorrentes do procedimento cirúrgico (náuseas, vômitos, anorexia, distensão abdominal); adequar a dieta quanto ao fornecimento de calorias e demais nutrientes essenciais; melhorar e/ou manter o estado nutricional do paciente; promover a sensação de bem-estar. Quando não há complicações, a realimentação oral é indicada nos primeiros cinco dias, o jejum transitório é perfeitamente tolerado pelo paciente e não requer terapêutica nutricional especial. Deve-se atentar para a hidratação e fornecer os principais eletrólitos, sódio e potássio (Baxter \& Waitzberg, 2006), pois o cólon é o seguimento de maior trânsito e sua ausência acentua a perda destas substâncias/nutrientes.

No pós-operatório tardio: adequar adieta ao hábito alimentar do paciente, visando ao seguimento domiciliar, após alta hospitalar; acompanhar o estado nutricional do paciente, visando prevenir alterações prejudiciais no mesmo (Baxter \& Waitzberg, 2006).

\section{REFERÊNCIAS}

BAXTER, Y.C.; WAITZBERG, D.L. Nutrição Oral nas Afecções Digestivas Cirúrgicas. In: WAITZBERG, D.L. Nutrição Oral, Enteral e Parenteral na Prática Clínica. $3^{\mathrm{a}}$ ed. São Paulo: Atheneu, 2006.

BERTARIO, L.; RUSSO, A.; SALA, P.; EBOLI, M.; GIAROLA, M.; D'AMICO, F. et al. Hereditary Colorectal Tumours Registry. Genotype and phenotype factors as determinants of desmoid tumors in patients with familial adenomatous polyposis. Int $\mathbf{J}$ Cancer, v. 95, p, 102-107, 2001.

CAMPOS, F.G.; HABR-GAMA, A.; KISS , D.R.; ATUI, F.C.; KATAYAMA, F.; GAMA-RODRIGUES, J. Manifestações extracolônicas da polipose adenomatosa familiar: incidência e impacto na evolução da doença. Arq Gastroenterol., v. 40, n. 2, p. 92-98, 2003. 
CAMPOS, F.G. Polipose adenomatosa familiar: revisão sobre as características clínicas, bases moleculares, tratamento cirúrgico e manuseio das manifestações extracolônicas. Gastroenterol Endosc Dig., v. 25, n. 2, p. 42-57, 2006.

GARCIA, C.G. GUTIERREZ, A.S.; BECERRA, E.A.; HERAS, B.S.; PLAZAS, J.G.; BROTONS, A.B. et al. Paciente con poliposis adenomatosa familiar y metástasis hepáticas de tumor neuroendócrino. Gastroenterol Hepatol., v. 34, n. 5, 329-32, 2011.

GARDNER, E.J. A genetic and clinical study of intestinal polyposis: a predisposing factor for carcinoma of the colon and rectum. Am J Genet., v. 3, p. 167-176, 1951.

HABR-GAMA, A.; CAMPOS, F.G. Síndromes polipóides do intestino grosso. In: PINOTTI, H.W. Tratado de clínica cirúrgica do aparelho digestivo. São Paulo: Atheneu; 1994.

KINZLER, K.W.; NILBERT, M.C.; SU, L.; SU, L.K.; VOGELSTEIN, B.; BRYAN, T.M. et al. Identification of FAP locus genes from chromosome 5q21. Science, v. 253, p. 661-4, 1991.

PENNA, C.; TIRET, E.; PARC, R.; SFAIRI, A.; KARTHEUSER, A.; HANNOUN, L. et al. Operation and abdominal desmoid tumors in familial adenomatous polyposis. Surg Gynecol Obstet., v. 177, p. 263-8, 1993.

ROSA, E.F.; COIMBRA, B.C. Câncer de cólon e estresse oxidativo. O Mundo da Saúde (CUSC), v. 33, n. 4, p. 415-418, 2009.

ZAGAR-SHOSTARI, K.; HILL, A.G. Optimization of perioperative care for colonic surgery: a review of the evidence. ANZ J Surg, v. 78, p. 13-23, 2008. 